\title{
FEBRE AMARELA: orientações de enfermagem à saúde dos viajantes em unidades básicas de saúdea
}

\author{
Anna Paula M A LLET ${ }^{b}$, Clarice M aria D A L L 'A G N OL c , Dirciara Barañano SOU ZA ${ }^{d}$
}

\section{RESUM 0}

E studo qualitativo, exploratório-descritivo, com objetivo de investigar as práticas de enfermagem para a orientação à saúde dos viajantes, tendo-se col etado dados junto a profissionais de enfer magem queatuam no setor de imunização de três U nidades Básicas de Saúde em Porto Alegre, Rio G rande do Sul, utilizando-se a técnica de entrevista semiestruturada. Da análise de conteúdo emergiram cinco categorias: perfil de atendimento, orientação à saúde, encaminhamentos para troca do Cartão $\mathrm{N}$ acional de Vacinação pelo Certificado Internacional de Vacinação, fonte de informações e material informativo. Os resultados sinalizam o início de uma organização das práticas de enfermagem voltadas para a saúde dos viajantes, indo além do enfoque na febre amarela. Falhas nas orientações para aquisição do Certificado Internacional de Vacinação ainda ocor rem e faltam materiais infor mativos. A pontase a necessidade de ampliar as discussões sobre a saúde dos viajantes para uma revisão das estratégias de organização desse cuidado e encaminhamentos para a construção de uma política específica.

D escritores: Educação em saúde. G estão em saúde. Saúde do viajante. Políticas públicas de saúde.

\section{RESUMEN}

E studio cualitativo, exploratorio-descriptivo, con objetivo de investigar las prácticas de enfer mería para la orientación a la salud de los viajantes, tras la recolección de datos junto a profesionales de enfermería que actúan en el sector de inmunización de tres U nidades Básicas de Salud en P or to A legre, Rio G rande do Sul, B rasil, utilizando la técnica de entrevista semiestructurada. A partir del análisis de contenido emergieron cinco categorías: perfil de atendimiento, orientación a la salud, encaminamientos para cambio de Carnet $\mathrm{N}$ acional de Vacunas por el Certificado I nternacional de Vacunas, fuente de infor maciones y material informativo. L os resultados señalan el principio de una organización delas prácticas de enfer mería volcadas hacia la salud de los viajantes, yendo más allá del enfoque en la fiebre amarilla. F allas en las orientaciones para la adquisición del Certificado Internacional de Vacunas aún ocur ren y faltan materiales informativos. Seapunta la necesidad de ampliar las discusiones sobre la salud de los viajantes para una revisión de las estrategias de or ganización de ese cuidado y encaminamientos para la construcción de una política específica.

Descriptores: E ducación en salud. G estión en salud. Salud del viajero. Políticas públicas de salud.

Titulo: F iebre amarilla: orientaciones de enfermería a la salud de los viajantes en unidades básicas de salud.

\section{ABST RACT}

T he objective of this qualitative, exploratory and descriptive study is to investigate the nursing practices related to travelers' health counseling. $D$ ata were coll lected from nursing professionals who work in the immunization sector of three $B$ asic $\mathrm{H}$ ealth U nits in P orto A legre, R io G rande do Sul, B razil, using thetechnique of semi-structured interview. F ive categories emerged from content analysis: careprofile, health orientation, referrals to exchangethe $\mathrm{N}$ ational I mmunization $\mathrm{Card}$ for the International Certificate of Vaccination, information sourceand infor mation material. T heresults signal the beginning of an or ganization of nursing practices focused on travelers' health, going beyond the focus on yellow fever. Failures in guidelines for acquisition of International Certificate of Vaccination still occur and information materials are missing. It points out to theneed of broadening thediscussion on travelers' health for a review of strategies for careorganization and refer rals for the construction of a specific policy.

D escriptors: $\mathrm{H}$ ealth education. $\mathrm{H}$ ealth management. T ravelers' health. $\mathrm{H}$ ealth public policy.

Title: Y ellow fever: nurse counseling on travelers' health at basic health clinics.

\footnotetext{
a Parte da monografia apresentada em dezembro de 2006 à E scola de Enfermagem da U niversidade Federal do Rio Grande do Sul (E E nfUFRGS).

' Enfermeira, D ocente do Curso de Enfermagem do Centro U niversitário do Planalto de Araxá (U NIARAXÁ), Coordenadora do G rupo de Estudos em U rgências e E mergências (GEUE), A raxá, M inas G erais, Brasil.

' D outora em Enfermagem, D ocente da E Enf-U FRG S, Coordenadora do Programa de Pós-G raduação da EE nf-U F RGS, Coordenadora do Núcleo de Estudos sobre G estão em Enfermagem (NEGE), Porto A legre, Rio G rande do Sul, Brasil.

- M estre em Enfermagem, D outoranda do Programa de Pós-G raduação em Enfermagem da EEnf-UFRGS, Bolsista REU NI, M embro do NEGE, Porto Alegre, Rio Grande do Sul, Brasil.
} 


\section{INT RODUÇÃO}

As evidências apresentadas pela ocorrência de epidemias e pandemias por doenças emergentes e reemergentes sinal izam nos viajantes um potencial para o transporte de agentes infecciosos, seus vetor es e ainda hábitos que podem propiciar a emergência de doenças. N o Brasil, embora a Secretaria de Vigilância em Saúde (SV S) e a A gência $\mathrm{N}$ acional de Vigilância Sanitária (AN VISA) venham impulsionando um movimento para a reordenação das práticas de cuidado à saúde dos viajantes, ainda se visualiza na oferta da vacina contra a febre amarela o principal elo entre essa população e os serviços de saúde ${ }^{(1)}$.

Enquanto possibilidade para esta situação associa-se o fato da vacina contra febre-amarela ser a única exigência para o trânsito internacional por áreas consider adas en dêmicas para a doença $a^{(2)}$. Além disso, as alterações do cenário epidemiológico da febre amarela no país, principal mente no Rio G rande do Sul (RS) - que até abril de 2009 teve 29 notificações de casos suspeitos de febre amarela silvestre e, destes, 18 casos foram confirmados para a doença, levando sete pessoas ao óbito(3) - já justificariam o interesse e a relevância da temática. E ssa realidade acena para o risco iminente de reurbanização da doença no Brasil, estando essa possibilidade de reintrodução da febre amarela nas cidades diretamente relacionada ao el evado número de pessoas não imunizadas que se deslocam rapidamente de e para áreas de risco(4).

As U nidades Básicas de Saúde (U BS) constituem-se em importantes campos a serem investigados. Isto por apresentarem-se como referência principal à oferta da vacina quando comparadas aos serviços de vacinação dos postos de controle sanitário da ANVISA ${ }^{(1,5)}$, ambos integrantes da rede de cuidado do viajante no Sistema Ú nico de Saúde (SU S). D iante disso e, por ser a equipe de enfermagem a responsável pelo serviço de imunização dessas U BS, surgiram alguns questionamentos: como a equipe de enfermagem das UBS de Porto A legre, referência para a vacina contra febre amarela, vem orientando o usuário-viajante que busca esse serviço? E stas equipes reconhecem a importância do espaço das U BS para a prática de educação em saúde? Existe integração entre as U BS e a AN VISA? Quem informa e atualiza esses profissionais de enfermagem para a prática de orientação a saúde dos viajantes e recomendação de vacinas a serem administradas?
N essa per spectiva, buscou-se investigar junto às U BS de Porto A legre, Rio Grande do Sul (RS), que imunizam contra febre amarela, sobre a prática de orientação de enfermagem à saúde dos usuários-viajantes. Como desdobramento deste eixo principal chegou-se a três objetivos específicos: identificar as fontes de informações dos participantes para a orientação aos usuários-viajantes; averiguar acerca do teor das informações que são concedidas pela equipe de enfermagem aos usuáriosviajantes sobre a exigência e/ ou recomendação da vacina contra febre amarela e a emissão do Certificado Internacional de Vacinação (CIV), bem como, outras recomendações à saúde dos viajantes, consider ando a área de destino dos mesmos; e sinalizar a representatividade do órgão regulador, A NVISA, junto à equipe de enfermagem nas questões relativas à vacinação contra febre amarela e o CIV. M enciona-se que após o período desta pesquisa, ano de 2006, o CIV passou por modificações em seu modelo, a fim de atender a nova versão do Regulamento Sanitário I nternacional, aprovada em 2005(6). Assim, encontra-se em fase de implantação no Brasil, desde junho de 2007, o Certificado Internacional de Vacinação e Profilaxia (CIVP).

\section{MÉT ODO}

A pesquisa situa-se na vertente qualitativa do tipo exploratório-descritiva. Esta modalidade proporciona maior familiaridade com o problema, possibilitando constituir hipóteses e descrever as características de determinada população ou fenômeno(7).

\section{Local de realização e sujeitos}

Como campo de pesquisa elegeu-se as UBS que a Secretaria M unicipal de Saúde (SM S) de Porto A legre/ RS indicou como locais de referência para a vacinação contra febre amarela, condição vigente até o término da coleta de dados. São elas: Centro de Saúde M odelo, Centro de Saúde I API e U nidade Básica de Saúde Tristeza, pertencentes respectivamente às $\mathrm{G}$ erências Centro, N oroeste/ H umaitá/ I lhas e Sul/ Centro Sul da SM S. Considera-se importante mencionar que, no segundo semestre de 2008, devido às mudanças no cenário epidemiológico da febre amarela no $\mathrm{RS}^{(3)}$, conforme referenciadas anteriormente, essa vacina passou a ser oferecida em todas as U BS do município de Porto Alegre, RS. 
A amostra de referência compõe-se de três profissionais da área de enfermagem, incluindo duas enfermeiras e um auxiliar de enfermagem, integ rantes de cada uma das três U BS em estudo. Priorizou-se como sujeito de estudo enfermeiras líderes de equipe por serem responsáveis por definirem micro-políticas para a organização e efetivação do trabal ho cotidiano, reper cutindo concretamente na qualidade das ações de saúde que são ofertadas aos usuários, como é o caso de viajantes que comparecem a esses locais em busca de vacinação contra a febre amarela e dos encaminhamentos pertinentes.

Foram excluídas aquel as enfermeiras que estavam ausentes no período da coleta de dados por motivos diver sos (licença de saúde, licença de interesse, férias, etc.) ou que não concordaram em participar quando da seleção. N esses casos, previa-se a indicação de outro infor mante junto ao responsável pelo serviço, o que de fato aconteceu em um dos três locais. A enfermeira responsável encontrava-se em licença saúde prolongada. 0 setor tomou a iniciativa de contatá-la por telefone e a mesma delegou um funcionário habilitado para conceder as informações, que no caso, foi um auxiliar de enfermagem.

\section{Coleta de dados e procedimentos de análise}

As entrevistas foram Semi-E struturadas, com gravação em áudio. Este tipo de entrevista caracteriza-se por utilizar um roteiro norteador, possibilitando que 0 entrevistado discorra sobre 0 tema proposto, sem condicionar respostas a determinadas alter nativas( ${ }^{(8)}$. Esta escolha, também, justifica-se por auxiliar nas pesquisas qualitativas ao propiciar aos integrantes da relação (pesquisadorpesquisado) oportunidade para darem alguma direção, a partir de um questionamento básico(9).

Para o tratamento das informações optou-se pela análise de conteúdo ${ }^{(8)}$, relacionando as estruturas semânticas (significantes) com as estruturas sociológicas (significados) dos enunciados. A pós transcrição na íntegra do material, proveniente das gravações em áudio, realizou-se a análise das entrevistas, conforme orientação das questões e objetivos do estudo.

\section{Precauções com aspectos éticos}

A tendendo às recomendações da Resolução 196/ 96 do M inistério da Saúde ${ }^{(10)}$, relativas a pes- quisas com seres humanos, os sujeitos do estudo foram informados sobre a natureza dessa investigação, objetivos, métodos, tempo de realização e benefícios previstos. Foi assegurado garantia de anonimato e de uso dos dados para fins únicos de pesquisa com orientação quanto ao caráter de livre participação. Também, se buscou anuência para gravação em áudio por meio de um T ermo de Consentimento Livre e Informado, entregue em duas vias, ficando uma em posse do entrevistado e outra em posse do pesquisador.

0 Projeto foi submetido à apreciação da Comissão de Pesquisa da Escola de Enfermagem da U niversidade Federal do Rio G rande do Sul com posterior envio para a Comissão de Ética em Pesquisa da Prefeitura M unicipal de Porto Alegre, onde foi homologado conforme Parecer no 86/ 06. Somente em posse dos documentos comprobatórios da homologação nessas instâncias procedeu-se à coleta de dados.

\section{APRESENT AÇÃO E DISCUSSÃO DOS RESULTADOS}

0 teor das informações foi agrupado em cinco categorias de análise: perfil de atendimento, orientações / recomendações à saúde, encaminhamentos para troca do Cartão Nacional de Vacinação (CNV) pelo CIV, fonte de informações e material informativo.

\section{Perfil de atendimento}

Embora as UBS selecionadas pela SM S do município de Porto Alegre, RS, para ofertarem a imunização contra a febre amarela tenham sido distribuídas geograficamente entre as gerências distritais Centro, N oroeste/ H umaitá/ I lhas e Sul/ Centro Sul com o objetivo de descentralizar a demanda, constatou-se neste estudo uma expressiva centralização da procura pela vacina contra febre amarela na gerência Centro. A partir da fala dos participantes da pesquisa, esta situação tem justificativa na localização privilegiada desse serviço e, portanto, pela facilidade de acesso, principalmente para aqueles usuários-viajantes oriundos de outros municípios da G rande Porto A legre, onde não há distribuição desta vacina. Porém, concorda-se com a defesa ${ }^{(11)}$ de que o uso de serviços pode ser uma medida de melhor acesso, mas não se explica apenas por ele. 
A despeito de 0 acesso ser um importante deter minante do uso, o uso efetivo dos serviços de saúde resulta de uma multiplicidade de fatores, tais como fatores individuais predisponentes, fatores contextuais e relativos à qualidade do cuidado, que influenciam o uso e a efetividade do cuidado. Assim, é importante que o serviço da gerência Centro seja analisado em suas particularidades, considerando-se sua demanda ser triplamente maior quando comparada aos serviços das duas outras gerências envolvidas no estudo.

Entretanto, não se pode deixar de associar a essa maior demanda o fato das agências de turismo da G rande Porto A legre serem a principal fonte de informação aos viajantes e terem essa gerência como referência para acesso à vacina contra febre amarel $a^{(5)}$. Com isso, visualiza-se a necessidade de desenvolver um planejamento, a fim de se estabelecer coordenação e integração entre as diferentes unidades de trabalho, rumo à organização dessa demanda.

0 atendimento ao usuário-viajante que busca o serviço de imunização, na maioria das vezes, é realizado pelos auxiliares de enfermagem. A enfermeira, na maioria das vezes, direciona sua atenção para atender suspeitas de reações adversas à vacina contra febre amarela, preenchendo o formulário de notificação específico para essas demandas. D essa forma, participa muito pouco na orientação dir eta ao usuário/ viajante. A penas numa das unidades a enfermeira relatou comparecer e permanecer mais tempo na sala de vacinas, disponibilizando-se a orientações gerais.

D e acordo com o teor das entrevistas, a participação do auxiliar de enfermagem como sujeito de estudo não acarretou divergências, comparada aos depoimentos das enfermeiras. Com isso observa-se uma integração enfermeira-auxiliar, evidenciando o trabalho em equipe já preconizado como essencial para a organização do serviço de enfermagem em estudos realizados na década de $50^{(12)}$.

\section{Recomendações e orientações à saúde}

Geralmente os usuários-viajantes procuram as U BS para receberem a vacina contra febre amarela. I sto justifica a ênfase dada pelos participantes às orientações referentes a esse imunobiológico, mencionando as contra-indicações da vacina contra febre amarela para gestantes, imunodeprimidos e pessoas alérgicas a proteína do ovo. Ressaltam, ainda, a validade de dez anos e a recomendação da aplicação a partir dos nove meses de idade. Para aqueles que estão recebendo a vacina pela primeira vez (primovacinação) são advertidas possíveis reações adver sas como: cefal éia leve, dor no corpo, dor no local da aplicação e hipertermia leve, variando conforme cada organismo. De acordo com o destino são fornecidas orientações relevantes quanto à prevenção de demais doenças transmissíveis. Reportam-se a importância do uso de repelente e roupas adequadas para proteção do corpo, a fim de evitar o contato com o vetor da doença, principalmente aquel es que se destinam as áreas de grande população de mosquitos, matas e rios.

A lém da vacina contra febre amarela, a equipe reconhece a necessidade de recomendar outras vacinas que não são obrigatórias aos viajantes, seguindo a orientação do $\mathrm{N}$ úcleo de I munizações da SM S. M encionam que a procura pela vacina contra a febre amarela, no serviço de imunização das U BS, coloca-os em papel de destaque pela oportunidade de contato direto com essa população. O portunidade essa que se constitui em espaço para a orientação sobre a necessidade de outros imunobiológicos, conforme seus roteir os de viagem e, sobre cuidados importantes durante a viagem para minimizar a exposição a riscos.

Sinal iza-se esse espaço como ideal para as práticas de educação em saúde, significando um trabal ho dirigido para atuar sobre o conhecimento das pessoas, a fim de desenvolver em senso crítico e capacidade de inter venção sobre suas vidas e sobre o ambiente com o qual interagem ${ }^{(13)}$.

Alguns viajantes em trânsito para áreas específicas são informados sobre a importância de serem vacinados contra poliomielite (VOP), de receberem a tríplice viral (M M R) e a antitetânica (dT ). Porém outras vacinas fazem parte da lista ${ }^{(14)}$ de imunobiológicos a serem consideradas durante o planejamento para o cuidado do viajante e que não foram mencionadas no estudo, como: a vacina contra o sarampo, hepatite A e B, encefal ite japonesa, tuberculose, influenza, entre outras. Algumas dessas vacinas, por não integ rarem o cal endário das vacinas de rotina do Programa Nacional de Imunizações (PN I $)^{(14)}$, não são oferecidas nas U BS. E sta situação culmina no encaminhamento do viajante pelo profissional de enfermagem para o Centro de Referência para I munobiológicos Especiais (CRIE). E ntretanto, é importante atentar-se para o fato de que este procedimento constitui-se numa prática 
informal e que muitas vezes não garante 0 acesso do viajante à vacina, uma vez que ainda não se têm um calendário específico para essa população. Diante disso, reforça-se a necessidade de uma política de saúde do viajante.

\section{Encaminhamentos para troca do CNV pelo CIV}

Nas U BS o registro da vacina contra febre amarela se dá no CNV, válido em todo território brasileiro. Se posteriormente, o viajante que dispõem deste registro nacional necessitar de comprovação da vacina no CIV, no caso de se deslocar para países que exigem essa vacinação de procedentes do Brasil, este é orientado pela equipe de enfermagem a procurar um serviço da ANVISA disponível em Postos de Vigilância Sanitária de Portos, A eroportos e Fronteiras distribuídos nos vinte e seis Estados e Distrito Federal, para que se efetue a transcrição dos dados.

V erificou-se com este estudo que existe a preocupação dos profissionais das U BS em esclarecer os acessos e documentos necessários aos viajantes com trânsito internacional para a obtenção do CIV junto aos serviços da A NVISA. Porém, esta orientação quanto à documentação necessária se dá de forma incompleta em dois dos três campos de pesquisa, culminando em entraves para a obtenção do CIV. Cartazes com informações sobre ender eço, tel efone e horários para troca foram observados em todos os locais do estudo, devido à necessidade de orientação aos viajantes com destino internacional. M as os participantes explicitam na fala, como dificuldade para o acesso dos viajantes ao CIV e para a confecção desses cartazes informativos, as freqüentes mudanças no horário de atendimento no Posto de Controle Sanitário da A N VISA, situado no Aeroporto Internacional Salgado Filho. Quanto ao local para troca do CN V pelo CIV, houve menção aos postos de atendimento da ANVISA situados no Cais do Porto de Porto Alegre e no A eroporto Internacional Salgado Filho, evidenciando-se nesteúltimo a referência para todos os três campos de pesquisa.

Para emissão do CIV a autoridade sanitária exige do viajante al guns procedimentos, entre el es: apresentação do CN V (com data, lote, carimbo, assinatura do profissional de saúde e identificação da unidade de saúde), apresentação do documento de identidade oficial com foto e a presença pessoal do viajante para assinatura do CIV ${ }^{(15)}$. N o entanto, essa documentação exigida está sendo informada de maneira incompleta, havendo apenas um dos postos com a orientação completa. A ssim, embora manifestem interesse em informar corretamente, na prática isto não se concretiza. Parece haver pouca sensibilização para a importância da temática que poderia estar sinalizando a necessidade de uma formação mais direcionada desses profissionais.

\section{Fonte de informações}

$N$ as falas de todos os entrevistados evidencia-se forte ancoragem ao N úcleo de I munizações da SM S, bem como a satisfação na oferta do suporte necessário às vacinas e atualizações periódicas de informações. 0 contato das unidades de saúde restringe-se ao $\mathrm{Núcleo,} \mathrm{especialmente} \mathrm{no} \mathrm{que} \mathrm{se}$ refere à lista dos países que exigem a vacinação contra a febre amarela de brasileiros e a lista dos locais dentro do país para os quais há uma recomendação desta vacina por tratar-se de áreas endêmicas ou de circulação do vírus. 0 N úcleo também se responsabiliza pelo repasse às U BS das orientações à saúde dos usuários-viajantes, de acordo com o destino dos mesmos. Ainda, articula-se com a SM S e esta com a Secretaria E stadual de Saúde (SE S), de forma regionalizada e hier arquizada, conforme preconizado pela Lei Orgânica da Saúde Lei 8.080/90 $90^{(16)}$, a qual estabelece as diretrizes do SU S. D esta forma, compete à SE S estabelecer um contato direto com a ANVISA, referida como M inistério da Saúde (M S) pelos sujeitos da pesquisa.

Periodicamente, o Núcleo de I munizações envia às U BS memorandos ou e-mails atualizando a lista dos países que exigem a vacinação contra a febre amarela, bem como as regiões brasileiras com áreas consideradas de risco. No entanto, foi possível identificar neste estudo que, o usuário-viajante quando acessa o ser viço de saúde está previamente orientado a solicitar a vacinação contra a febre amarela, sendo as agências de viagem as principais fontes de informação desses usuários. T al fato justifica a importância de se estabelecer uma parceria entre as UBS e essas agências, com vistas a um trabal ho integ rado que culmine na implementação de estratégias para o cuidado e orientação aos viajantes.

\section{Material informativo}

Com esta investigação verificou-se que na Rede Básica de Saúde do município de Porto Alegre não há distribuição de material informativo com 
orientações sobre a vacinação contra a febre amarela ou outros imunobiológicos recomendados a viajantes, orientações relacionadas a cuidados durante a viagem e encaminhamentos para aquisição do CI V. Q uando questionado aos informantes desse estudo sobre sugestões de conteúdo, considerando a possibilidade de se elaborar um material para distribuição aos usuários-viajantes nas UBS, evidenciou-se uma expressiva empolgação em seus relatos.

Os temas sugeridos foram quase que unânimes entre todos os sujeitos, destacando-se o conceito de febre amarela. M odo de transmissão; como se pega? M edidas preventivas e outros cuidados; como posso me prevenir? Sintomas da doença (febre amarela); o que se sente? Lista dos países que exigem a vacina e regiões recomendadas. Orientações/ recomendações de quem pode ou não receber a vacina; posso receber a vacina contra a febre amarela? Reações adversas; 0 que posso sentir após a administração da vacina? Prazo entre a data da vacinação e o embarque; quantos dias antes da viagem devo fazer a vacina?

E ste resultado sinal iza uma contradição instalada. E mbora a importância de orientar os viajantes sobre a necessidade de outros imunológicos tenha sido mencionada pelos participantes, a temática saúde do viajante ainda encontra-se fortemente ancorada na idéia da vacinação contra a febre amarela.

\section{CONSIDERAÇÕES FINAIS}

A convergência das informações entre os sujeitos das três U nidades que disponibilizam a vacinação contra a febre amarela sinal izam o início de uma organização para a prática de orientação à saúde dos usuários-viajantes, pela enfermagem. Entretanto percebe-se um envolvimento ainda muito discreto das enfermeiras com a temática. A demanda excessiva na UBS da gerência Centro incita a necessidade de uma melhor avaliação das particularidades dessa U nidade, a fim de se investigar possíveis difer enças na oferta desse cuidado com relação às outras duas U BS envolvidas na pesquisa.

Por outro lado, o fácil acesso também aparece na fala dos participantes como justificativa para a maior procura desse serviço. A o pensar sobre esse acesso, percebe-se uma centralização pré-estabelecida da demanda por se identificar que somente três U BS no município de Porto A legre ofereciam a vacina contra febre amarela, afunilando os caminhos de acesso. Convém mencionar que a noção de ter ritorialidade trazida pelo SU $S^{(16)}$, preconiza a não centralização da demanda. E ssa situação somente se reverteu diante da emergência apresentada pelo cenário epidemiológico da febre amarela no E stado do Rio G rande do Sul a partir do ano de 2008, quando essa vacina passou a ser oferecida em todas as U BS.

No trabalho em equipe, verificou-se interação dos profissionais na prática comunicativa, bem como, na realização das tarefas pertinentes a imunização. Os profissionais das U BS estudadas demonstraram que existe uma integração da equipe e uma val orização do trabal ho grupal, tanto pelas enfermeiras quanto pelos auxiliares de enfermagem. Os participantes reconhecem a necessidade de recomendar aos usuários-viajantes outros imunobiológicos, al ém da vacina contra a febre amarela, considerando o curso da viagem. Porém, verifica-se uma contradição ao solicitarem a elaboração de material informativo contemplando somente temas referentes à febre amarela.

No que se refere à fonte de informações, o N úcleo de I munizações da SM S de Porto Alegre/ RS obteve grande destaque na fala dos participantes, evidenciando-se a credibilidade dessa coordenadoria para a Rede Básica de Saúde. Quanto ao órgão regulador das práticas de orientação e controle sanitário de viajantes, AN VISA, verificou-se que o mesmo tem pouca representatividade junto às $U B S$, no que diz respeito às ações rel acionadas com a vacina contra febre amarela, fato que também se evidenciou em pesquisa(5) realizada com as Agências de T urismo. Quanto às orientações para a troca do CN V pelo CIV, os profissionais de enfermagem reforçaram nas falas o compromisso em orientar corretamente sobre as indicações e contraindicações da vacina contra a febre amarela e a indicação da mesma de acordo com o país de destino do viajante. $M$ esmo assim, ainda se percebe al gumas falhas que parecem estar relacionadas com a pouca comunicação entre as U BS e os Postos de Controle Sanitário da AN VISA, local que for nece o CIV.

D estaca-se a importância desse estudo ao dar visibilidade à temática saúde dos viajantes e a necessidade de se discutir a organização desse cuidado na enfermagem. Os ser viços de imunização, que integram às UBS, aparecem como espaços privilegiados para um repensar dessa prática, visto receber os usuários-viajantes que na maioria das vezes 
buscam a vacina contra febre amarela. Os resultados, também apontam para a necessidade de uma maior integração entre a ANVISA e a Secretaria de Vigilância em Saúde, por representarem as instâncias gover namentais responsáveis pela atenção à saúde do viajante no SU S, sem perder de vista a integ ração com outros órgãos e organizações que tenham interface com a temática.

Sugere-se, com o resultado desse estudo, um repensar voltado para um planejamento sistêmico, partindo de uma análise situacional, focalizando as forças inter nas que agem na organização do serviço e, por fim, verificando as influências de âmbito externo. Por fim, recomenda-se a ampliação desse debate entre os gestores federais, estaduais e municipais e profissionais da saúde para a revisão das estratégias de gestão no âmbito desse cuidado, sinalizando a importância de uma política específica.

\section{REFERÊNCIAS}

1 Dall'Agnol CM, Souza D B, M allet AP, Nunes PRS, Liberali J. 0 (des)conhecimento dos viajantes sobre a exigência da vacinação contra febre amarela: um estudo no A eroporto I nternacional de Porto A legre, RS. REM E : Rev M in Enferm. 2007;11(4):375-80.

2 World Health Organization. Vaccine-preventable diseases, vaccines and vaccination [ Internet] . G eneva; 2009 [ cited $2009 \mathrm{~N}$ ov 04] . Available from: www. who.int/ topics/ immunization/ es.

3 Secretaria da Saúde (RS), Centro Estadual de Vigilância em Saúde. Situação atual dos casos suspeitos de febre amarela [ Internet] . Porto Aleg re; 2009 [ citado 2009 abr 22] . D isponível em: http:/ / www.saude. rs.gov.br/ wsa/ portal/ index.jsp?menu=servicos\& $\operatorname{cod}=22246 \mathrm{f}$.

$4 \mathrm{~W}$ orld $\mathrm{H}$ ealth Organization. Assessment of yellow fever epidemic risk: a decision-making tool for preventive immunization campaigns. W kly Epidemiol Rec. 2007;82(18):153-60.

$5 \mathrm{M}$ allet AP. O rientação à saúde dos viajantes: 0 papel da Rede Básica de Saúde em Porto Alegre [ monografia] . Porto Alegre: Escola de Enfermagem, U niversidade Federal do R io G rande do Sul; 2006.

\section{Endereço da autora / Dirección del autor / \\ Author's address:}

Anna Paula M allet

Rua G eralda Rufino Borges, 235,

Veredos da Cidade

38182-224, Araxá, M G

E-mail: annarmallet@yahoo.com.br
6 World Health Organization. International Health Regulations (2005) [ I nternet] . G eneva; 2008 [ cited 2009 N ov 04] . Available from: http:/ / www.who.int/ ihr/ en/index.html.

7 Gil AC. Como elaborar projetos de pesquisa. 4a ed. São Paulo: A tlas; 2002.

8 Bardin L. A nálise de conteúdo. Lisboa: E dições 70; 1977.

9 T urato ER. T ratado da metodologia clínico-qualitativa. Petrópolis: Vozes; 2003.

10 M inistério da Saúde (BR ), Consel ho N acional de Saúde. Resolução 196, de 10 de outubro de 1996: diretrizes e nor mas técnicas regulamentadoras de pesquisa envolvendo seres humanos. Brasília (D F ); 1996.

11 T ravassos C, M artins M . U ma revisão sobre os conceitos de acesso e utilização de serviços de saúde. $C$ ad Saúde Pública. 2004;20 Supl 2:5190-8.

12 Peduzzi M, Ciampone M HT. T rabalho em equipe e processo grupal. In: Kurcgant $\mathrm{P}$, organizadora. G erenciamento em enfer magem. Rio de Janeiro: G uanabara Koogan; 2005. p. 108-24.

13 Cecagno D, Siqueira HCH, Vaz M RC. Falando sobre pesquisa, educação e saúde na enfermagem. Rev G aúcha E nferm. 2005;26(2):154-60.

14 Lo CS, M ascheretti M, Chaves T SS, L opes M H. Vacinação dos viajantes: experiência do A mbulatório dos Viajantes do Hospital das Clinicas da Faculdade de M edicina da U niversidade de São Paulo. Rev Soc Bras M ed T rop. 2008;41(5):474-8.

15 M inistério da Saúde (BR), A gência N acional de Vigilância Sanitária. O rientação ao viajante [ Internet] . Brasília (DF); 2006 [ citado 2006 maio 05] . Disponível em: http:// www.anvisa.gov.br/ paf/ viajantes/ index.htm.

16 M inistério da Saúde (BR ). L ei no 8.080, de 19 de setembro de 1990: dispõe sobre as condições para a promoção, proteção e recuper ação da saúde, a organização e o funcionamento dos serviços correspondentes e dá outras providências [ Internet]. Brasília (DF); 1990 [ citado 2009 nov 04] . Disponível em: http:/ / portal.saude.gov.br/ portal/ arquivos/ pdf/ LE I 8080. pdf.

Recebido em: 20/ 11/ 2009

A provado em: 12/ 05/ 2010 